\title{
Patients' Perception of Pain During Implant Placement Using Inferior Alveolar Nerve Block Anesthesia Versus Infiltration Anesthesia: A Randomized, Split-mouth, Double-Blinded, Original Prospective Clinical Trial
} Article

\author{
Omar N. El-Prince, Ahmed Wallan AlAhmary
}

Department of Oral and Maxillofacial Surgery, Al-Farabi College for Nursing and Dentistry, Riyadh, Saudia Arabia

\begin{abstract}
Purpose: This study aimed to assess patients' perception of pain towards placing dental implants in mandibular region using inferior alveolar nerve block versus infiltration anesthesia.

Materials and Methods: 50 patients who needed implant placement in the posterior mandibular region bilaterally were included in this study. Two equal groups were assigned for this study, Group A: where inferior alveolar nerve block (IANB) was used for implant placement and Group B: where Supraperiosteal infiltration was used. Patients' perception of pain, age, gender, distance of each implant to mandibular canal, duration of surgery, and number of implants placed on each mandibular side were all recorded and statistically analyzed.

Results: No significant difference between pain during drilling for dental implants in both groups was observed, however, pain during implant placement was higher in group B. correlation was found between implant placement and distance to mandibular canal in group B. Pain perception during implant preparation and placement increased with the presence of adjacent teeth.

Conclusion: Both infiltration and IANB can be used for drilling and placement of implants. Using a Lidocaine infiltration anesthesia might give the operator an alert about the inferior alveolar nerve (IAN) approximation during only implant placement as nerve injury can be encountered during drilling due to absence of alerting pain. It is anticipated the patient's pain perception might increase with the increased number of teeth adjacent to the site of implant.
\end{abstract}

Key Words: Dental implant, mandibular infiltration anesthesia, nerve block, pain

Received: 24 September 2020, Accepted: 14 October 2020

Corresponding Author: Omar N. El-Prince, Department of Oral and Maxillofacial Surgery, Al-Farabi College for Nursing and Dentistry, Riyadh, Saudia Arabia, Tel.: +966595026978, E-mail: oelprince@alfarbi.edu.sa

ISSN: 2090-097X, October 2020, Vol. 11, No. 4

\section{INTRODUCTION}

Administration of local anesthesia is the most common procedure in the dental practice ${ }^{[1]}$. Profound local anesthesia is considered as part and parcel in every successful dental procedure that might cause pain ${ }^{[2]}$. This can be attributed to the fact that not only does the profound anesthesia facilitate the dental procedures by decreasing the patient's sense of pain but also it is one of the important parameters for patient when choosing a dentis $\mathrm{t}^{[3]}$. Additionally, poor control of pain might interfere with appropriate dental managements ${ }^{[4]}$.

Inferior alveolar nerve block (IANB) is more commonly used than infiltration anesthetic technique in performing different restorative and dentoalveolar surgeries in the mandibular region. This can be explained by the increased thickness of the buccal cortical plate of bone in this area which hinders the dissemination of the anesthetic solution through infiltration technique ${ }^{[5]}$. However some studies have suggested that the use of infiltration technique, with an anesthetic agents which have a superior diffused properties such as Articaine, might give similar results in reducing pain like IANB during dental procedures ${ }^{[6]}$.

When it comes to dental implant placement as one of the dentoalveolar surgical procedures, some articles have suggested that using an infiltration technique during implant drilling and placement in mandibular region may enable the patient to sense the pain when there is an approximation to the $\operatorname{IAN}^{[7]}$. On the contrary, other researchers has found that there is no correlation between the pain perceived during drilling for implant placement and approximation to the $\operatorname{IAN}^{[8,13]}$.

Pain arise from different dental implant procedure is notoriously volatile owing to the multifactorial nature of pain perceived, which differs significantly from one patient to another ${ }^{[9]}$. This study was intended to be designed in a split mouth design to remove lots of inter-individual 
variability that might encounter the researchers during assessment of pain ${ }^{[10]}$.

The aim of this study was to assess the patient's perceptions of pain during implant placement and implant drilling separately using inferior alveolar nerve block anesthesia versus infiltration anesthesia. Moreover, to assess the patient's perception of pain when approximating the mandibular canal during implant placement using inferior alveolar nerve block anesthesia versus infiltration anesthesia.

\section{PATIENTS AND METHODS}

Pre-surgical phase: This study was carried out as a randomized double blinded split-mouth clinical trial. The qualified patients were selected from the outpatient clinic of Alfarabi Colleges of Dentistry and Nursing.

This research was performed following the recommendations of the Consort Statement for reporting RCTs and the ethical principles of the Helsinki Declaration regarding research on humans.

The study was approved by the ethical committee and informed consent was obtained from all patients. A list of inclusion and exclusion criteria were made to qualify patients for this study. Inclusion criteria was: patient age $>18$ years, patients who have mandibular bilateral posterior edentulous space, while exclusion criteria was: medically compromised patients that have a medical condition affects the implant surgery, Any contraindication for implant surgery, patients who can distinguish between IAN and infiltration anesthesia (e.g: dentists), two consecutive failed anesthesia, patients who needed complicated surgical procedure for implant placement (e.g: guided bone regeneration), patients who have any painful or neurological disorders.

All patients were specifically informed that the main objective was to provide painless surgery, and that if they felt pain, immediate anesthesia reinforcement would be provided.

Clinical examination, evaluation of local and systemic factors, panoramic radiograph and or CBCT scan were used to plan surgery.

In order to prevent the possible effect of the order of the anesthesia on the patient's evaluation of the anesthetic method, block anesthesia and implant placement or infiltration anesthesia with implant placement in the one side were performed after over 3 weeks of the surgery in the other side.

Surgical Procedure: Anesthesia technique was administrated for all patients in both groups by a dentist who was involved in the study, in the absence of the surgeon to blind both the surgeon and the patient.

The choice of anesthetic technique for each patient's mandibular side was randomized by tossing a coin.

The anesthetic drug used was $2 \% 1.8 \mathrm{~mL}$ Lidocaine with 1:100,000 epinephrine (Alexandria pharmaceutical company, Alexandria, Egypt)

In group A: IANB was performed with accordance to Halstead approach ${ }^{[11]}$. After the IANB was confirmed a supplemental buccal infiltration was administered for the buccal nerve. In group B: Supraperiosteal mandibular infiltration technique was administered and $0.3 \mathrm{ml}$ under the lingual periosteum to help to raise the flap ${ }^{[7]}$.

After 5 minutes of latent period if anesthesia was not achieved after two approaches, the patient received intervention was excluded from the analysis. After anesthesia was confirmed, the operator entered the room and began the surgery. All surgical interventions consisted of raising a muco-periosteal flap, drilling with copious cooling at 800 - $1200 \mathrm{rpm}$, manual and instrumental implant placement (Dentium Co., Ltd. Seoul, korya), and primary closure of the wound.

All patients were instructed to warn the surgeon in the case of pain sensation and to describe the pain felt on the visual analogue scale (VAS) which consists of a $10 \mathrm{~cm}$ line to evaluate the amount of pain, ranging from 0 (no pain whatsoever) to 10 (worst pain imaginable) ${ }^{[15]}$.

The blinded surgeon recorded the patient's perception of pain and reported to the dentist who was involved in the study.

After completion of the implant surgery patients' implants position were assessed radiographically via digital panoramic view and the distance between each implant apex and mandibular canal (MC) was recorded. (Figure 1)

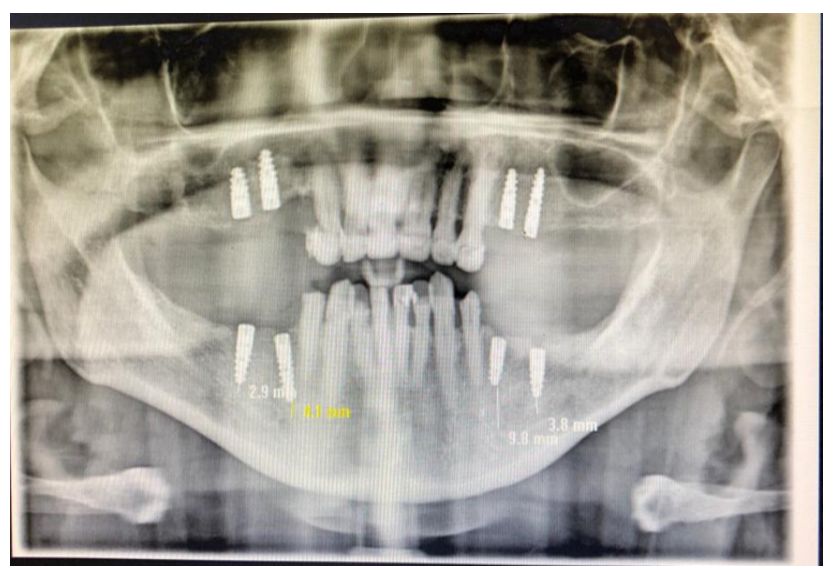

Fig. 1: distance between implant apex and mandibular canal (MC) 
Outcome measures: Patients' population: regarding number of participants, age, sex , number of natural teeth adjacent to implant site and number of implants placed in every group were recorded and statistically analyzed.

Patients' perception of maximum pain during drilling for implant was recorded in Visual Analogue Scale (VAS).

Patients' perception of maximum pain during implant placement was recorded in Visual Analogue Scale (VAS).

Distance between implant apex and MC: was measured and recorded in millimeter $(\mathrm{mm})$ using Trident software program (Trident S.r.l. Via Artigiani, 425014 Castenedolo (BS), Italy). These measurements were taken by a single blinded investigator who was not involved in the clinical trial.

Duration of surgery was measured in minutes from the time of placing the incision to the last suture placed.
All data were fed to the computer and analyzed using IBM SPSS software package version 20.0. (Armonk, NY: IBM Corp). The Kolmogorov- Smirnov test was used to verify the normality of distribution of variables, Mann Whitney test was used to compare between two groups for not normally distributed quantitative variables, Kruskal Wallis test was used to compare different groups for not normally distributed quantitative variables and followed by Post Hoc test (Dunn's) for pairwise comparison. Spearman coefficient was used to correlate between quantitative variables. Significance of the obtained results was judged at the $5 \%$ level.

\section{RESULTS}

Patients' population: 50 patients were included in this study (27 males and 23 females). Mean age for the whole participants was 44 (SD 11) years. No patient withdrawal was recoded. (Figure 2)

\section{CONSORT}

TRANSPARENT REPORTING Of TRIALS

\section{CONSORT 2010 Flow Diagram}

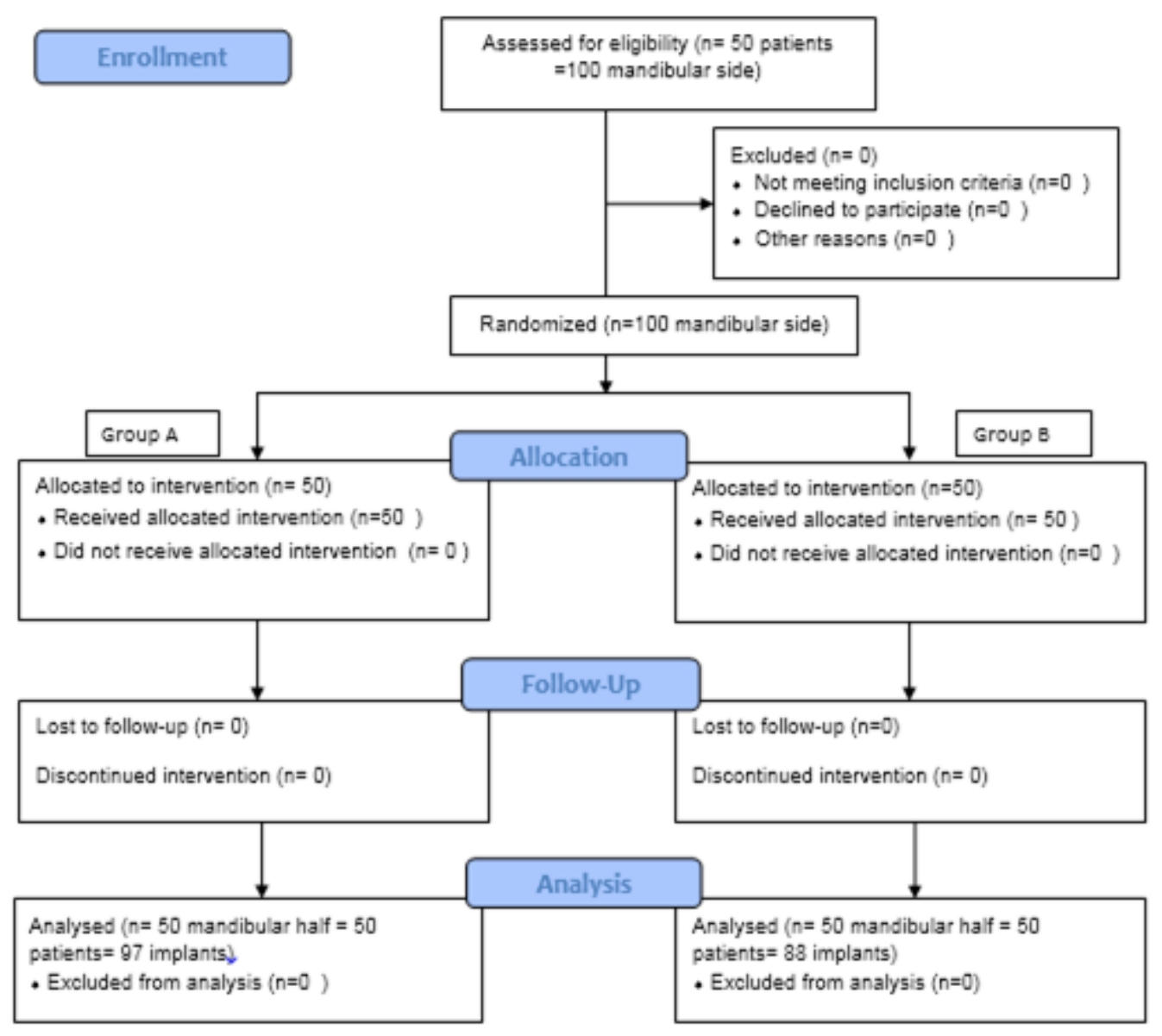

Fig. 2: Consort flaw chart 
The total number of implants placed was 185 implants (97 implants in group A and 88 implants in group B). In group A: number of implants placed between two adjacent teeth was 17 , number of implants placed adjacent to one tooth was 25 , and number of implants placed in edentulous area was 55 . While In group B: number of implants placed between two adjacent teeth was 13, number of implants placed adjacent to one tooth was 28 , and number of implants placed in edentulous area was 47 .

Patients' perception of pain: No correlation was found between VAS values and age $(p=0.58)$ or gender $(p=0.32)$, or tooth to be replaced $(p=0.73)$, in the whole population

In both groups, the maximum pain recorded (VAS) during implant placement was significantly higher than that recorded during implant drilling $(p<0.05)$, However there was no significant difference between pain recorded (VAS) during drilling for implants in both groups $(p=0.707)$. (Table1).

Table 1: Comparison between the two studied groups according to pain recorded during drilling and during implant placement

\begin{tabular}{lcccc}
\hline $\begin{array}{l}\text { Pain recorded } \\
\text { during }\end{array}$ & $\begin{array}{c}\text { Group A } \\
(\mathrm{n}=96)\end{array}$ & $\begin{array}{c}\text { Group B } \\
(\mathrm{n}=87)\end{array}$ & $\mathrm{U}$ & $p$ \\
\hline Drilling & & & \\
& & & \\
Min. - Max. & $0.0-3.0$ & $0.0-3.0$ & & \\
Mean \pm SD. & $0.84 \pm 0.93$ & $0.90 \pm 0.95$ & 4050.0 & 0.707 \\
Median & 1.0 & 1.0 & & \\
& & & \\
Implant & & & \\
placement & $0.0-5.0$ & $0.0-6.0$ & & \\
& $1.60 \pm 1.16$ & $2.22 \pm 1.51$ & $3241.5^{*}$ & $0.007^{*}$ \\
Min. - Max. & 1.0 & 2.0 & \\
Mean \pm SD. & &
\end{tabular}

U: Mann Whitney test

$\mathrm{p}$ : $p$ value for comparing between the studied groups

*: Statistically significant at $p \leq 0.05$

In both groups, there was a weak negative correlation between maximum pain recorded during drilling and distance from the mandibular canal (in group $\mathrm{Ar}_{\mathrm{s}}=-0.304$ and in group $\mathrm{B} r_{s}=-0.353$ ), however there was a strong negative correlation between the distance to mandibular canal and maximum pain recorded during implant placement in group B with infiltration anesthesia $r_{s=}-0.669$ Figure 3 ( $a$ and $b$ ).

In both groups there was also significant association between duration of surgery and pain during drilling or implant placement $(p<0.05)$.

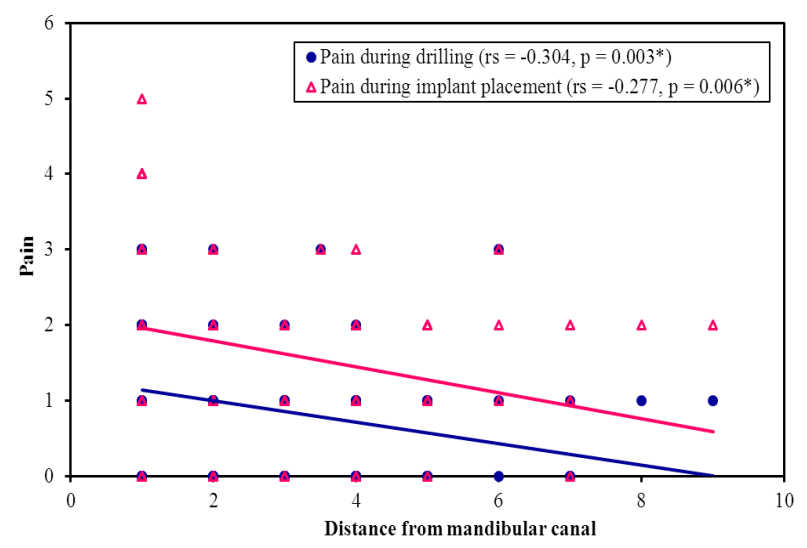

Fig. 3 (a): Correlation between distance from mandibular canal with pain during drilling and pain during implant placement in Group A

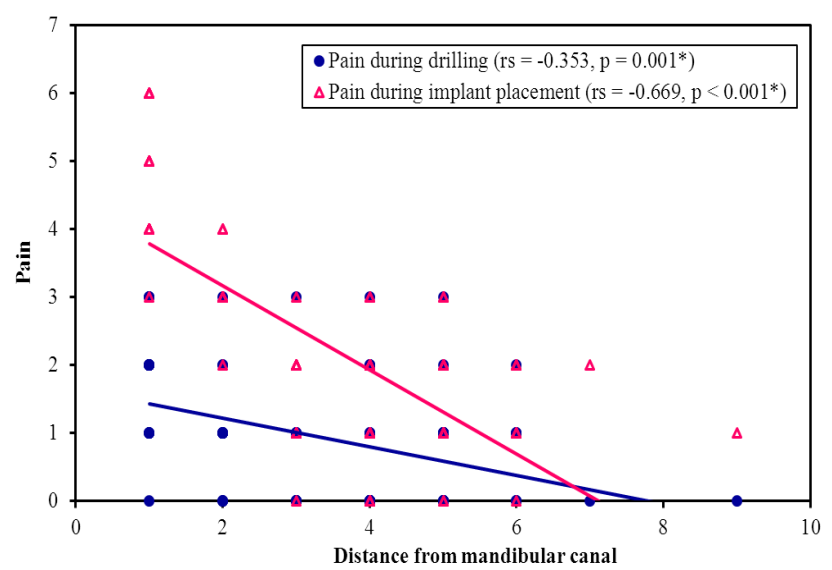

Fig. 3 (b): Correlation between distance from mandibular canal with pain during drilling and pain during implant placement in Group B

In both groups, there was a significant correlation between the increase in pain during implant drilling and placement and presence of adjacent teeth to implant site. Additionally, the most pain recorded was during implant placement adjacent to two teeth in group B (mean $=4.85 \pm 0.90$ ) and the least pain recorded was during implant drilling in the absence of adjacent teeth in group A $($ mean $=0.26 \pm 0.45)($ Table 2$)$ 
Table 2: Relation between presence of teeth and pain recorded during implant placement or implant drilling in both groups

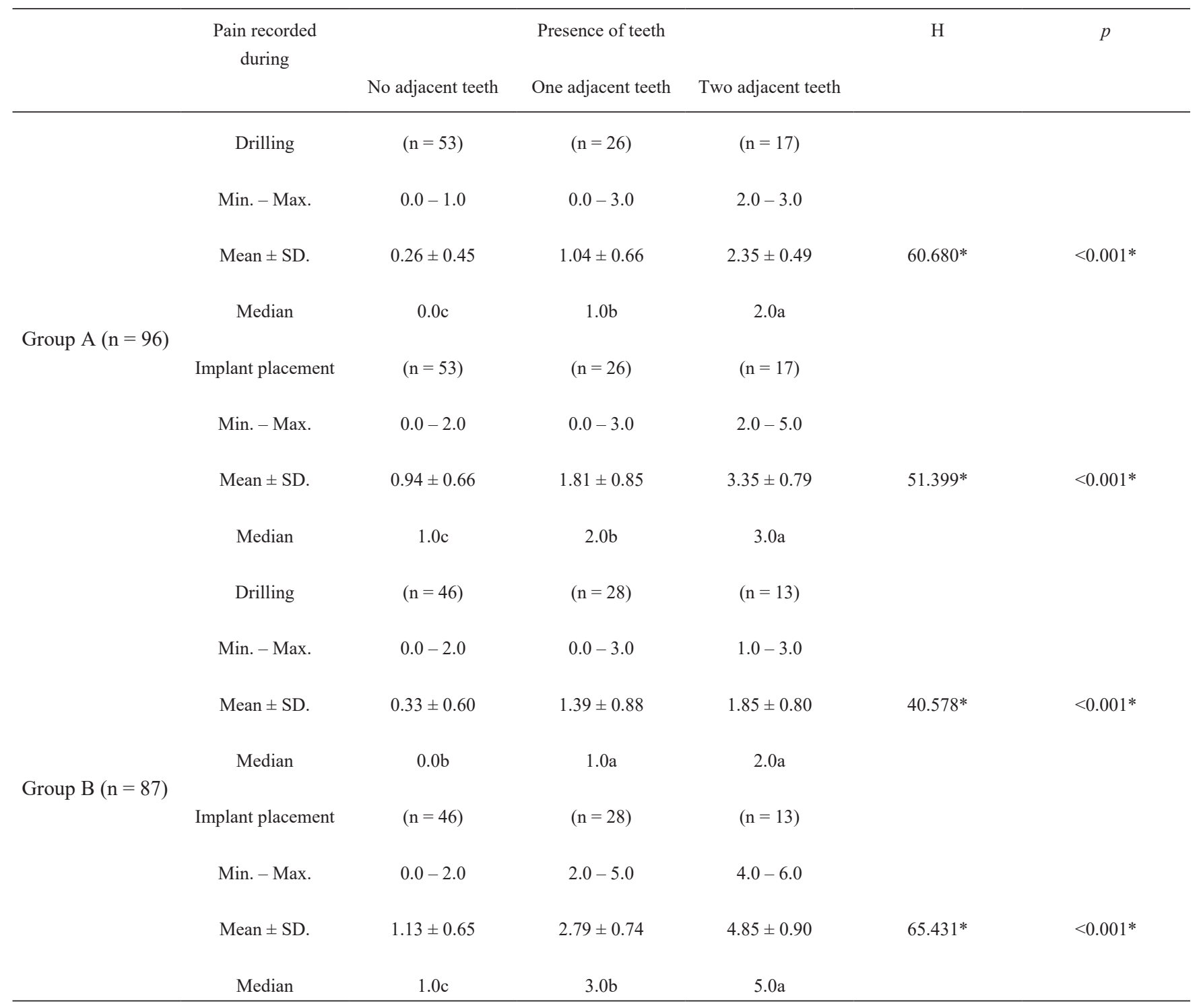

Means in the same raw with common small letters are not significant (i.e. Means with Different letters are significant)

$\mathrm{H}$ : $\mathrm{H}$ for Kruskal Wallis test, Pairwise comparison bet. each 2 groups was done using Post Hoc Test (Dunn's for multiple comparisons test)

$\mathrm{p}: p$ value for comparing between the studied groups

*: Statistically significant at $p \leq 0.05$

\section{DISCUSSION}

Several factors are contributed to perception of pain in the dental practice during different dental or dentoalveolar surgical procedures ${ }^{[12]}$. Accordingly this study was designed in a split mouth fashion to remove most of the interindividual variability that might encounter the assessor when trying to evaluate a multifactorial aspect like pain ${ }^{[10]}$.

This research has also compared the patient's pain perception using two anesthetic technique for dental implant placement. While the administration of inferior alveolar nerve block ensures pain control during dental implant placement, many studies have reported that the same procedure can be done effectively with only Supraperiosteal infiltration anesthesia ${ }^{[7,8,13,19]}$.
In this research the mean VAS value for implant drilling in group A was $0.84 \pm 0.93$ while in group B was $0.90 \pm 0.95$. These results were in accordance with a clinical trial done by Garcia-Blanco $\mathrm{M}$ et al. in $2018^{[8]}$, however the aforementioned study did not distinguish between the pain during drilling and pain during implant placement. In this study pain during drilling was significantly lower than that perceived during implant placement (mean VAS for implant placement was $1.60 \pm 1.16$ in group A and $2.22 \pm 1.51$ in group B).

Pain during implant placement in group B was significantly higher than that reported in group A with IANB, same results has been reported from study done by Garcia-Blanco $\mathrm{M}$ et al. in $2018^{[8]}$ and Etoz OA et al. 
in $2011^{[13]}$ which reported that pain perceived during implant surgery in infiltration group was higher than that reported in IANB group.

This study has found weak correlation between distance from the mandibular canal and pain during drilling in both groups, yet, there was a strong negative correlation between pain during implant placement and approximation to IAN in group B $\left(r_{s}=-0.669\right)$. In other words, in group B with infiltration anesthesia, pain during implant placement increased when the distance to mandibular canal decreased. Heller et al. in $2011^{[7]}$ has concluded that giving infiltration anesthesia during implant placement might alert the surgeon about the possibility of nerve injury by increased pain perceived from the patient when approximating the IAN. On the contrary, Garcia-Blanco M. et al. ${ }^{[8]}$ and Etoz OA et $a l^{[13]}$ have reported that no correlation is found between giving infiltration anesthesia and pain during implant placement. The difference in results reported can be attributed to the use of different types of local anesthetic drug, such as Articaine in Garcia-Blanco M. et al..$^{[8]}$ and Etoz OA et al. ${ }^{[13]}$ which has a superior diffusion properties than Lidocaine which is used on this study ${ }^{[14]}$. Additionally, although Sánchez-Siles in 2016 ${ }^{[19]}$ used Articaine infiltration anesthesia to place posterior mandibular implants, he has concluded that giving a small dose of infiltration anesthesia that doesn't necessarily anesthetize the lip will be efficient in warning the surgeon when approximation to IAN occurs.

In the current study, it was evident that pain has increased when number of teeth adjacent to implant increases. Same observation has been mentioned in the clinical trial conducted by Garcia-Blanco M. et al. ${ }^{\left[{ }^{[1}\right.}$. These findings can be attributed to the mechanical stimulation of nerve endings of the periodontal ligament surrounding the adjacent teeth $^{[16]}$.

Many studies have reported that there is a correlation between increase in pain perception and duration of surgery ${ }^{[8,17,18]}$, same observation has been noted in the current study where a positive correlation between increased duration of surgery and increase in VAS measures was reported in both groups $\left(r_{s}=.698\right)$.

\section{CONCLUSION}

From this study it can be concluded that both infiltration and IANB can be used for drilling and placement of dental implants. However, using a Lidocaine infiltration anesthesia might give the operator an alert about the IAN approximation during only implant placement as nerve injury can still be encountered due to absence of alerting pain during drilling for implant. It is anticipated the patients' pain perception might increase with the increase number of teeth adjacent to the site of implant.

\section{CONFLICT OF INTEREST}

There are no conflicts of interests.

\section{REFERENCES}

1. Uckan S, Cilasun U, Erkman O. Rare ocular and cutaneous com $\neg$ plication of inferior alveolar nerve block. J Oral Maxillofac Surg. 2006;64:719-21.

2. Majid OW, Ahmed AM: The anesthetic efficacy of articaine and lidocaine in equivalent doses as buccal and non-palatal infiltration for maxillary molar extraction: A randomized, doubleblinded, placebo-controlled clinical trial. J Oral Maxillofac Surg 76:737, 2018

3. Narang R, Mittal L, Saha S, Aggarwal VP, Sood P, Mehra S. Empathy among dental students: A systematic review of literature. J Indian Soc Pedod Prev Dent. 2019;37(4):316-326. doi:10.4103/JISPPD.JISPPD_72_19

4. TellezM,PotterCM,KinnerDG, et al. Computerized Tool to Manage Dental Anxiety: A Randomized Clinical Trial. J Dent Res. 2015;94(9 Suppl): 174S-80S. doi:10.1177/0022034515598134

5. Venkat Narayanan J, Gurram P, Krishnan $\mathrm{R}$, Muthusubramanian V, Sadesh Kannan V. Infiltrative local anesthesia with articaine is equally as effective as inferior alveolar nerve block with lidocaine for the removal of erupted molars. Oral Maxillofac Surg. 2017;21(3):295299. doi:10.1007/s10006-017-0628-z

6. Rathi NV, Khatri AA, Agrawal AG, M SB, Thosar NR, Deolia SG. Anesthetic Efficacy of Buccal Infiltration Articaine versus Lidocaine for Extraction of Primary Molar Teeth. Anesth Prog. 2019;66(1):3-7. doi:10.2344/anpr-65-04-02

7. Heller AA, Shankland WE $2^{\text {nd }}$. Alternative to the inferior alveo $\neg$ lar nerve block anesthesia when placing mandibular dental implants posterior to the mental foramen. J Oral Implantol. 2001;27:127-33.

8. Garcia-Blanco M, Gualtieri AF, Puia SA. A randomized controlled trial comparing nerve block and mandibular infiltration techniques in posterior mandible implant surgeries. J Clin Exp Dent. 2018;10(10):e1003-e1010. Published 2018 Oct 1. doi:10.4317/jced.54330

9. Porporatti AL, Bonjardim LR, StuginskiBarbosa J, Bonfante EA, Costa YM, Rodrigues Conti PC. Pain from Dental Implant Placement, 
Inflammatory Pulpitis Pain, and Neuropathic Pain Present Different Somatosensory Profiles. J Oral Facial Pain Headache. 2017;31(1):19-29. doi:10.11607/ofph.1680

10. Lesaffre E, Philstrom B, Needleman I, Worthington $\mathrm{H}$. The design and analysis of split-mouth studies: what statisticians and clinicians should know. Stat Med. 2009;28(28):3470-3482. doi:10.1002/ $\operatorname{sim} .3634$

11. Montserrat-Bosch M, Figueiredo R, NogueiraMagalhães $\mathrm{P}$, Ar $\neg$ nabat-Dominguez J, ValmasedaCastellón E, Gay-Escoda C. Efficacy and complications associated with a modified inferior alveolar nerve block technique. A randomized, triple-blind clinical trial. Med Oral Patol Oral Cir Bucal. 2014;19:391-7.

12. Maslamani M, Sedeqi F. Antibiotic and Analgesic Prescription Patterns among Dentists or Management of Dental Pain and Infection during Endodontic Treatment. Med Princ Pract. 2018;27(1):66-72. doi:10.1159/000486416

13. Etoz OA, Er N, Demirbas AE. Is supraperiosteal infiltration anesthesia safe enough to prevent inferior alveolar nerve during posterior mandibular implant surgery?. Med Oral Patol Oral Cir Bucal. 2011;16(3):e386-e389. Published 2011 May 1. doi:10.4317/medoral.16.e386

14. Yilmaz K, Tunga U, Ozyurek T. Buccal infiltration versus inferior alveolar nerve block in mandibular $2^{\text {nd }}$ premolars with irreversible pulpitis. Niger J Clin Pract. 2018;21(4):473-477. doi:10.4103/ njcp.njcp_135_17

15. Serrano-Atero MS, Caballero J, Ca-as A, García-Saura PL, Serra $\neg$ no-Álvarez C, Prieto J. Valoracion del dolor (II). Rev Soc Esp Dolor. 2002;9:109-121.

16. Ken Y, Tachikawa N, Kasugai S. Differences in knowledge related to dental implants between patients with and without a treatment history of dental implants. Clin Oral Implants Res. 2017;28(9):1062-1066. doi:10.1111/clr.12918

17. Reissmann DR, Poxleitner P, Heydecke G. Location, intensity, and experience of pain after intra-oral versus extra-oral bone graft harvesting for dental implants. J Dent. 2018;79:102-106. doi:10.1016/j.jdent.2018.10.011

18. Srinivasan M, Meyer S, Mombelli A, Müller F. Dental implants in the elderly population: a systematic review and meta-analysis. Clin Oral Implants Res. 2017;28(8):920-930. doi:10.1111/ clr. 12898

19. Sánchez-Siles M, Camacho-Alonso F, SalazarSánchez N, Aguinaga-Ontoso E, Muñoz JG, Calvo-Guirado JL. A low dose of subperiosteal anaesthesia injection versus a high dose of infiltration anaesthesia to minimise the risk of nerve damage at implant placement: A randomised controlled trial. Eur J Oral Implantol. 2016;9(1):59-66 